\title{
Evaluations of neuromuscular dynamics of hyperactive reflexes poststroke
}

\author{
Jie Liu, BS; ${ }^{1-3}$ Dali Xu, PhD; ${ }^{1,4}$ Yupeng Ren, MS; ${ }^{1}$ Li-Qun Zhang, PhD ${ }^{1-2,5 *}$ \\ ${ }^{1}$ Rehabilitation Institute of Chicago, Chicago, IL; ${ }^{2}$ Department of Biomedical Engineering, Northwestern University, \\ Evanston, IL; ${ }^{3}$ College of Engineering, Huazhong Agricultural University, Wuhan, China; ${ }^{4}$ Department of Kinesiol- \\ ogy, Western Illinois University, Macomb, IL; ${ }^{5}$ Departments of Physical Medicine and Rehabilitation and Orthopaedic \\ Surgery, Northwestern University, Evanston, IL
}

\begin{abstract}
Tendon reflexes are widely used in clinics to conveniently evaluate various neurological disorders. This study characterized neuromuscular dynamics of tendon reflexes at the elbow with multiple quantitative measures in both patients with stroke and nondisabled controls. We employed a handheld instrumented hammer to tap the triceps muscle tendon at various elbow flexion angles and measured the tapping force, triceps electromyography, and elbow extension torque to characterize neuromuscular dynamics of tendon reflexes quantitatively in terms of the tendon reflex gain, contraction rate, half relaxation rate, reflex loop delay, and reflex threshold in tapping force. We found that the tendon reflex gain, contraction rate, and half relaxation rate were significantly higher while the reflex threshold was significantly lower across different joint angles in patients with stroke than in nondisabled controls $(p<0.05)$, indicating hyperactive neurological state and muscle contraction dynamics poststroke. This study presents a convenient and quantitative method to evaluate reflex excitability and muscle contraction dynamics.
\end{abstract}

Key words: contraction rate, half relaxation rate, neurological disorder, reflex gain, reflex loop delay, reflex threshold, rehabilitation, spasticity, stroke, tendon reflex.

\section{INTRODUCTION}

Stroke is the leading cause of adult disability and the third leading cause of death in the United States, with 730,000 strokes occurring each year [1-2]. Spasticity is a complicated clinical symptom, characterized by a velocity- dependent increase in tonic stretch reflexes (muscle tone) with exaggerated tendon jerks, resulting from hyperexcitability of the stretch reflex [3]. Spasticity is a major source of disability in many patients with brain or spinal cord injuries of many different etiologies, including stroke, traumatic spinal cord injury, multiple sclerosis, and cerebral palsy [3]. The hypertonus and reflex hyperexcitability associated with spasticity disrupt the remaining functional use of muscles, impede motion, and may cause severe pain. Prolonged spasticity may be accompanied by structural changes in muscle fibers and connective tissue, which may reduce joint range of motion and lead to clinical contracture. Many therapeutic paradigms, such as antispastic medication [4-5], physical modalities [6-7], botulinum toxin injection [8-9], intrathecal baclofen pumps [10], and novel surgical techniques [11], were developed and applied for reducing spasticity and improving function. Even though such techniques can benefit patients, their effects on spasticity have not been well quantified because accurate and convenient measures of hyperactive reflexes that are suitable for clinical practice are lacking [12].

\footnotetext{
Abbreviations: ANOVA = analysis of variance, EMG = electromyography, $f_{\text {th }}=$ threshold in tapping force, $G_{s}=$ tendon reflex gain, $R_{c}=$ contraction rate, $R_{h r}=$ half relaxation rate, $t_{d}=$ reflex loop delay.

* Address all correspondence to Li-Qun Zhang, PhD; Rehabilitation Institute of Chicago, 345 E Superior St, Room 1406, Chicago, IL 60611; 312-238-4767; fax: 312-238-2208. Email: 1-zhang@northwestern.edu

DOI:10.1682/JRRD.2010.04.0065
} 
Mechanisms underlying spasticity are still not clearly understood. The increased resistance to passive movement in a spastic limb can be caused by of nonreflex changes like contracture as well as reflexive changes like hyperactive reflexes [13-14]. Reflex and nonreflex mediated contributions to the increased resistance must be separated to evaluate and understand the mechanisms underlying spasticity. Furthermore, it is not clear whether the hyperactive reflexes associated with spasticity are caused by an increase in tendon reflex gain $\left(G_{S}\right)$ [15] or a decrease in reflex threshold [16-17]. For assessing the severity of the spastic state and identifying its key characteristics poststroke, evaluating hyperactive reflexes quantitatively in terms of $G_{S}$ and reflex threshold in people with stroke is important.

The purposes of this study were investigating the mechanisms underlying spasticity in stroke and quantitatively evaluating hyperactive reflexes. Our hypothesis is that hypereflexia and spasticity poststroke are associated with significantly increased $G_{s}$, increased contraction rate $\left(R_{C}\right)$, and decreased reflex threshold. We performed the experiment isometrically at the elbow joint to both minimize nonreflex contributions and manifest and characterize hyperactive reflexes accurately.

\section{METHODS}

\section{Subject Selection}

Ten patients with chronic stroke and elbow impairment (age: $51.9 \pm 8.3$ yr [mean \pm standard deviation], height: $171.3 \pm 4.5 \mathrm{~cm}$, weight: $76.6 \pm 15.8 \mathrm{~kg}, 6$ male and 4 female) and eleven nondisabled controls with no prior history of neurological disorders (age: $43.1 \pm 10.9$ yr, height: $171.6 \pm 6.6 \mathrm{~cm}$, weight: $66.7 \pm 7.3 \mathrm{~kg}, 10$ male and 1 female) participated in the study. We examined each patient with stroke at the beginning of the experiment using the clinical tendon reflex scale ranging from 0 to 4 , with $0=$ no response, $1=$ low average, $2=$ average normal, $3=$ brisker than average, and $4=$ hyperactive and association with clonus. The patients with stroke were not using antispastic medication during the study.

\section{Experimental Procedures}

Each subject sat upright with their trunk strapped to the seat backrest and shoulder abducted $80^{\circ}$ and flexed $10^{\circ}$ (Figure 1). We cast and mounted the distal forearm, wrist, and proximal hand onto the distal end of an alumi-

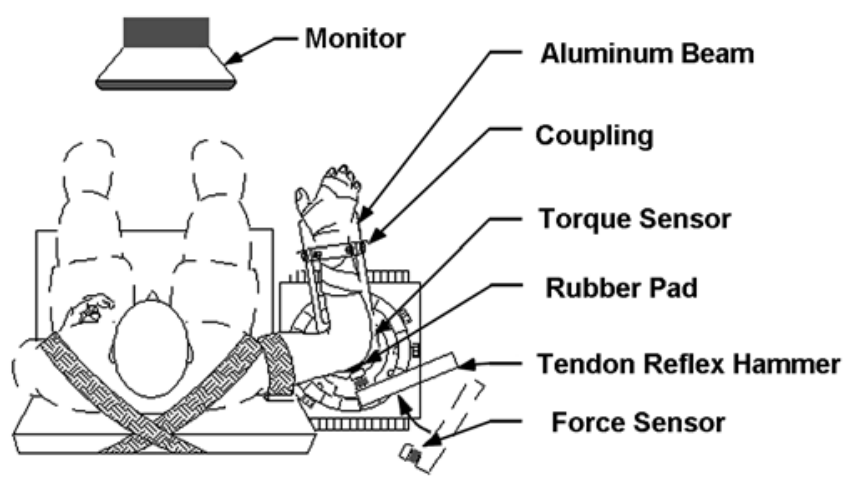

Figure 1.

Experimental setup for evaluating tendon reflexes at elbow joint. Motor is mounted on supporting frame with motor shaft aligned with elbow flexion axis. Torque sensor is mounted between motor shaft and aluminum beam to measure reflex elbow extension torque. Cast is fixed to aluminum beam through coupling. Motor is fixed at selected joint flexion angle to keep elbow at isometric condition. Rubber pad is mounted at most sensitive spot on triceps tendon, which is tapped with instrumented tendon hammer with force sensor mounted at its tip.

num beam and mounted the other end of the beam onto a motor shaft through a torque sensor that measured the elbow extension torque. We aligned the elbow flexionextension axis with the motor shaft and locked the motor at the selected elbow flexion angle during tendon taps, restricting the elbow at an isometric condition.

Using a traditional tendon reflex mallet, we located the most sensitive spot on the triceps tendon with the strongest reflex response. We pressed a $10 \mathrm{~mm}$-diameter hemisphere self-adhesive rubber pad onto the triceps tendon at the most sensitive spot. We used an instrumented tendon hammer with a force sensor mounted at its head to tap the rubber pad. The flat impact surface of the instrumented tendon hammer hit the dome-shaped rubber pad, which made the tapping force transmission onto the tendon more accurate and consistent, reducing variations of the tendon reflexes [10].

During the experiment, the subject was seated comfortably and asked to fully relax and not react to or anticipate the taps. If the subject felt inclined to move or change the posture, we would wait until he or she relaxed again. At the beginning, we adjusted the tapping force so that a triceps muscle contraction was clearly evoked. We then tapped the triceps tendon at approximately that level about seven times during a trial, with a random interval averaging about $2.5 \mathrm{~s}$. We collected three trials at each 
joint angle and repeated the tapping at $60^{\circ}, 90^{\circ}$, and $120^{\circ}$ elbow flexion. We sampled the tendon tapping force, triceps electromyography (EMG) signals, and elbow joint extension torque at $500 \mathrm{~Hz}$ after low-pass filtering (8thorder Butterworth filter at $230 \mathrm{~Hz}$ cutoff).

\section{Data Processing}

The sampled EMG signals were full-wave rectified. We filtered the tendon tapping force, rectified EMG, and elbow extension torque signals digitally using a finite impulse response low-pass filter with a cutoff frequency of $150 \mathrm{~Hz}$. The signals were then processed interactively. We inspected the elbow extension torque and triceps EMG signals to see whether any random voluntary contraction or marked noise occurred. If so, the relevant taps would not be selected. We then segmented the force, EMG, and torque signals into multiple taps, aligned by the tapping force peak moment. Each data segment was about $670 \mathrm{~ms}$ long, starting from $70 \mathrm{~ms}$ before the tapping force peak and ending $600 \mathrm{~ms}$ after the peak.

\section{System Impulse Response}

Because the reflex torque is induced by the tendon tapping force and varied with the tapping force, treating them as output and input of the tendon reflex system, respectively, is appropriate [10]. We used the system impulse response to characterize the reflex torque as the output of a system excited by the tendon tapping force. We identified the impulse response (the input-output relationship) from the experimental data as follows: because the tapping force was rather brief, it could be approximated as a pulse. Therefore, we approximated the impulse response as the reflex torque response scaled by the area of the corresponding tapping force pulse [11].

\section{Parameters Characterizing Tendon Reflex Dynamics}

We used several physiologically meaningful parameters to characterize the impulse response of the tendon reflex system (Figure 2). Within a certain range, the $G_{s}$ reflex torque varied with the tendon tapping force in that a stronger tapping force elicited a stronger reflex torque. In system analysis, $G_{S}$ was the gain measure of the tendon reflex system at zero frequency, calculated as the area of the impulse response.

$R_{C}$ characterized the slope of the ascending segment of the impulse response (calculated over period from onset to peak instant of impulse response). $R_{C}$ characterized the muscle contraction dynamics with a unit of in

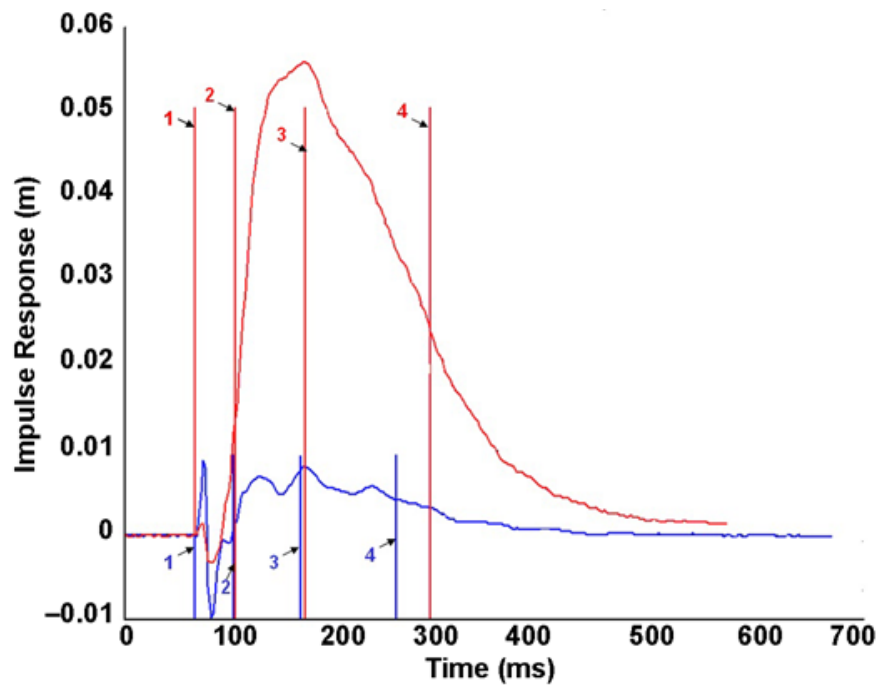

Figure 2.

Representative impulse responses of tendon reflex from patient with stroke (red line) and nondisabled control (blue line). Vertical lines correspond to (1) start of tapping impact, (2) onset of reflex-mediated torque response, (3) peak torque response, and (4) 50\% of peak response. Notice that spike after line 1 was because of tappinginduced mechanical impact transmitted to torque sensor.

meters per second. Similarly, the half relaxation rate $\left(R_{h r}\right)$ characterized the slope of the descending segment of the impulse response, the dynamic rate of muscle relaxation (over period from peak instant of impulse response to instant of $50 \%$ of peak).

In addition to the above parameters characterizing the system impulse response, we used the peak tendon tapping force that was used to elicit tendon reflexes for each subject to characterize the reflex threshold in tapping force $\left(f_{t h}\right)$, which characterizes the reflex excitability. Finally, we characterized the reflex loop delay $\left(t_{d}\right)$ quantitatively as the delay from the start of the tapping force to the onset of the reflex-mediated torque response, which was also shown in the impulse response (Figure 2).

\section{Statistical Analysis}

We used the Student $t$-test to determine whether the $G_{s}, R_{c}, R_{h r}, f_{t h}$, and $t_{d}$ were different between the patients with stroke and nondisabled controls. We compared $G_{s}$, $R_{c}, R_{h r}, f_{t h}$, and $t_{d}$ between the two different subject groups across the three different elbow joint angles using the repeated measures analysis of variance (ANOVA) 
$(2 \times 3$, two different groups and three different joint angles). We set the significance level at $\alpha=0.05$.

\section{RESULTS}

The patients with stroke showed considerable spasticity and hyperactive reflex as indicated by the clinical scales. Their clinical tendon reflex scale score was $3.0 \pm$ 0.6 and their Modified Ashworth Scale [4,7-8] score (ranging from 0 to 4 ) was evaluated as $2.3 \pm 1.1$. They were $8.4 \pm 4.6$ yr poststroke.

\section{Typical Tendon Reflexes in Patients with Stroke and Nondisabled Controls}

Compared with the nondisabled controls, patients with stroke had significantly different neuromuscular dynamics in tendon reflexes. As shown in the representative cases (Figure 3), the threshold in tapping force for the patients with stroke (12 $\pm 2 \mathrm{~N})$ was much lower than that for the nondisabled controls $(41 \pm 4 \mathrm{~N})(p=0.01)$. On the other hand, the reflex-mediated EMG response and elbow extension torque in the patients with stroke
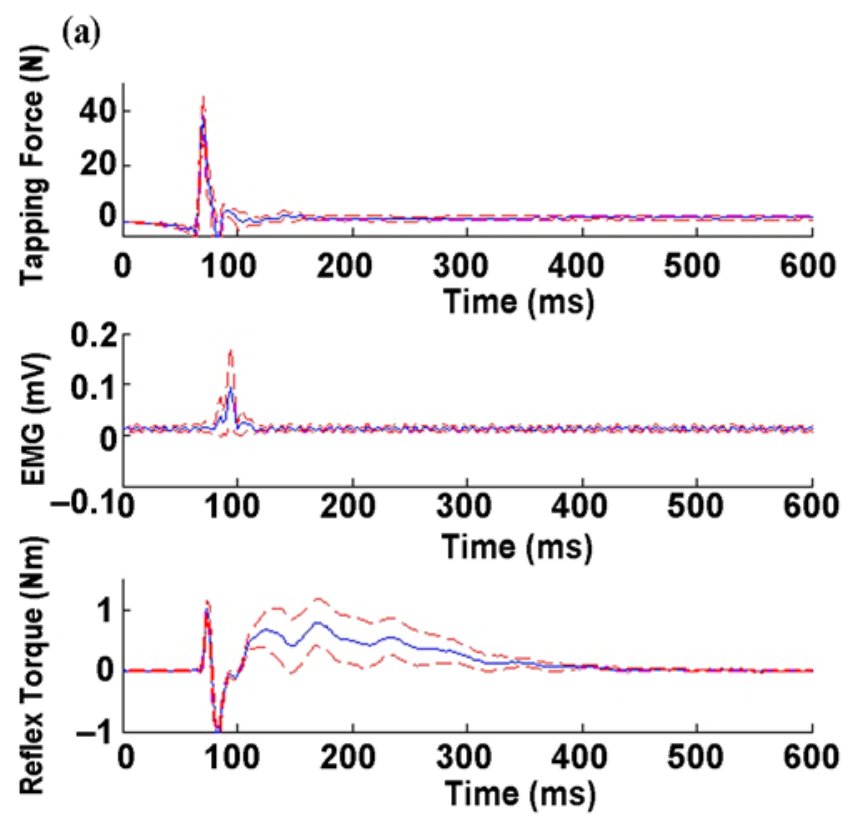

were much higher and changed much quicker than their counterparts in the nondisabled controls.

\section{Impulse Response of Tendon Reflexes}

The impulse responses characterized the dynamic relationship between the tapping force and reflex-mediated torque response in system analysis (Figure 2). Compared with the nondisabled controls, the spastic elbow showed a much stronger tendon reflex impulse response with much higher amplitude and quicker increased amplitude, indicating stronger and quicker reflex responses associated with hyperactive reflexes.

\section{Tendon Reflex Gain}

The $G_{S}$ in patients with stroke was much higher than that in nondisabled controls (Figure 4(a)). The $G_{S}$ of the patients with stroke was $0.66 \pm 0.66 \mathrm{~m} \cdot \mathrm{ms}, 2.52 \pm$ $1.55 \mathrm{~m} \cdot \mathrm{ms}$, and $3.13 \pm 2.15 \mathrm{~m} \cdot \mathrm{ms}$ at $60^{\circ}, 90^{\circ}$, and $120^{\circ}$ elbow flexions, respectively. In contrast, the $G_{s}$ of the nondisabled controls was $0.23 \pm 0.19 \mathrm{~m} \cdot \mathrm{ms}, 0.59 \pm$ $0.28 \mathrm{~m} \cdot \mathrm{ms}$, and $0.51 \pm 0.25 \mathrm{~m} \cdot \mathrm{ms}$ at $60^{\circ}, 90^{\circ}$, and $120^{\circ}$ elbow flexions, respectively.

ANOVA with repeated measures showed that the $G_{s}$ of the patients with stroke was significantly higher than
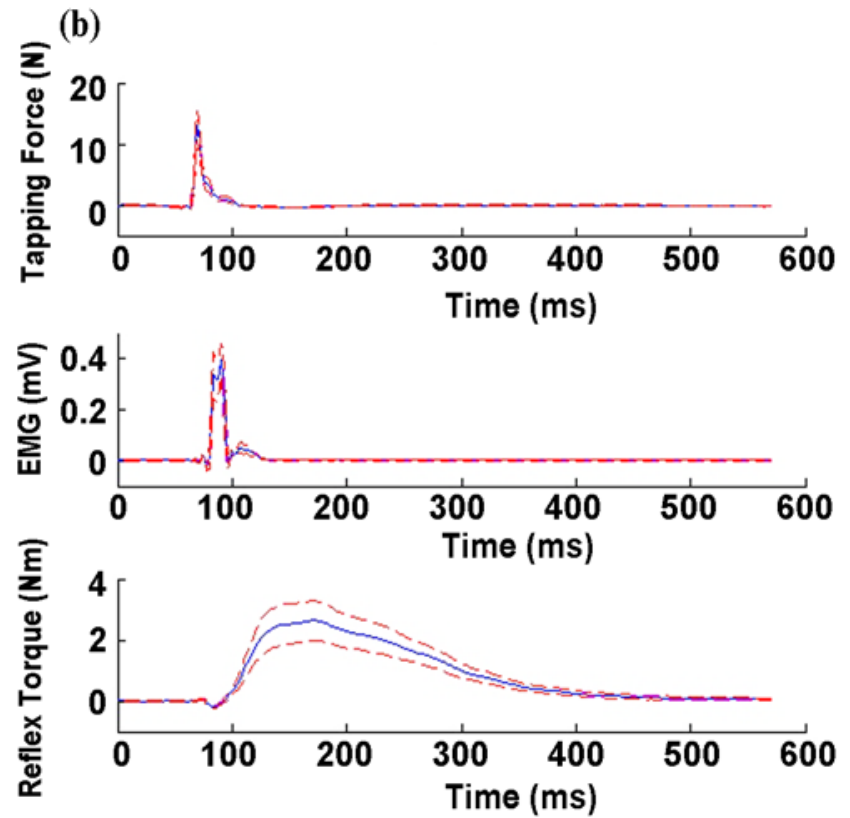

Figure 3.

Representative tendon tapping results over multiple taps of triceps tendon with elbow joint at $90^{\circ}$ flexion in (a) nondisabled controls and (b) patients with stroke. Blue solid lines and red dashed lines represent mean \pm standard deviation, respectively. EMG = electromyography. 

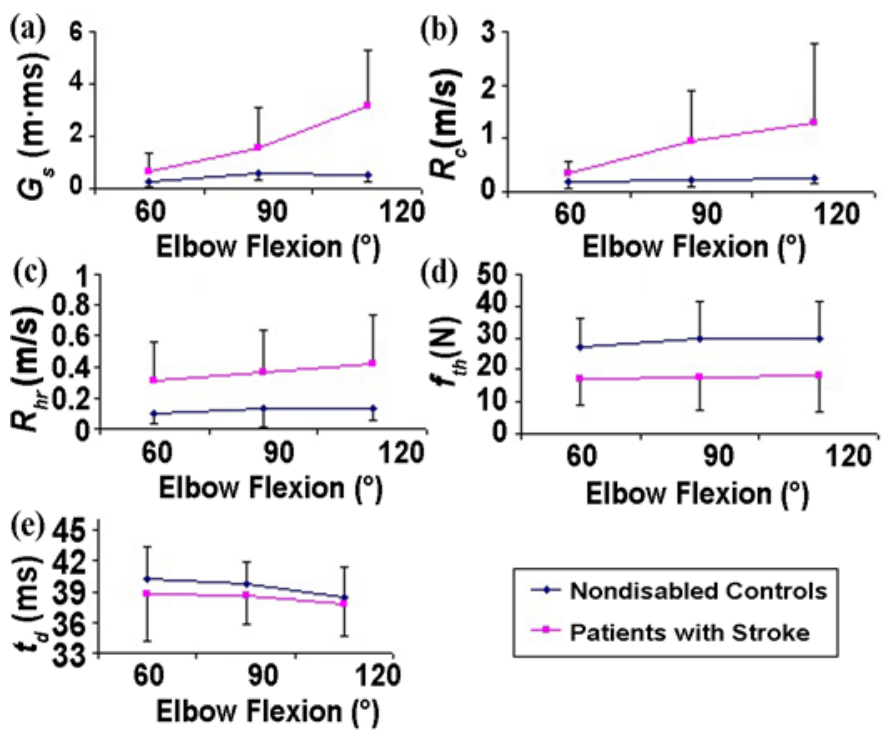

Figure 4.

Comparison of (a) tendon reflex gain $\left(G_{S}\right)$, (b) contraction rates $\left(R_{c}\right)$, (c) half relaxation rate $\left(R_{h r}\right)$, (d) reflex threshold in peak tendon tapping force $\left(f_{t h}\right)$, and (e) reflex-loop delay $\left(t_{d}\right)$ between patients with stroke and nondisabled controls across three elbow flexion angles. Vertical bars represent standard deviation across subjects.

that of the nondisabled controls across the different angles $\left(F_{1,7}=54.895\right.$ and $\left.p=0.01\right)$. There also was a significant difference across the different angles in the patients with stroke $\left(F_{2,6}=10.185\right.$ and $\left.p=0.01\right)$, but there was no effect of interaction between the subject groups and angles.

\section{Tendon Reflex Contraction Rate and Half Relaxation Rate}

The ANOVA procedure with repeated measures showed that the tendon reflex $R_{C}$ of patients with stroke was significantly higher than that of the nondisabled controls across the different angles $\left(F_{1,7}=7.123, p=0.03\right)$. The $R_{C}$ of patients with stroke versus nondisabled controls at $60^{\circ}, 90^{\circ}$, and $120^{\circ}$ elbow flexions were $0.34 \pm 0.2$ versus $0.15 \pm 0.10 \mathrm{~m} / \mathrm{s}, 0.96 \pm 0.94$ versus $0.20 \pm 0.10 \mathrm{~m} / \mathrm{s}$, and $1.29 \pm 1.48$ versus $0.15 \pm 0.10 \mathrm{~m} / \mathrm{s}$, respectively (Figure 4(b)).

Similarly, the $R_{h r}$ of the patients with stroke was significantly higher than that of nondisabled controls $\left(F_{1,7}=\right.$ 14.014, $p=0.01$ ) (Figure 4(c)). The $R_{h r}$ of the patients with stroke versus nondisabled controls at $60^{\circ}, 90^{\circ}$, and $120^{\circ}$ elbow flexions were $0.32 \pm 0.26$ versus $0.078 \pm 0.04$ $\mathrm{m} / \mathrm{s}, 0.33 \pm 0.29$ versus $0.09 \pm 0.05 \mathrm{~m} / \mathrm{s}$, and $0.41 \pm 0.31$ versus $0.11 \pm 0.06 \mathrm{~m} / \mathrm{s}$, respectively.

\section{Reflex Threshold in Tapping Force Between Patients with Stroke and Nondisabled Controls}

The threshold in tapping force in the spastic limbs in patients with stroke was significantly lower than that in nondisabled controls $\left(F_{1,7}=18.017, p=0.01\right)$ across all different elbow joint angles. The $f_{\text {th }}$ for the patients with stroke versus nondisabled controls at $60^{\circ}, 90^{\circ}$, and $120^{\circ}$ elbow flexions were $16.5 \pm 8.4$ versus $28.3 \pm 8.3 \mathrm{~N}, 18.0 \pm$ 11.1 versus $30.7 \pm 11.6 \mathrm{~N}$, and $17.9 \pm 10.9$ versus $31.3 \pm$ $10.9 \mathrm{~N}$, respectively, indicating more reduced reflex threshold and more increased reflex excitability in the patients with stroke than in the nondisabled controls (Figure 4(d)). Furthermore, no difference existed in the threshold in tapping force across different joint angles in either of the two groups. This indicates that the threshold is not sensitive to joint angle change in the patients with stroke and nondisabled controls. On the other hand, the manually delivered taps were not controlled as precisely as a servomotor controlled tapping, which may also have contributed to the insignificant dependence on joint angle.

\section{Reflex Loop Delay}

The mean values of the $t_{d}$ for the patients with stroke versus nondisabled controls at $60^{\circ}, 90^{\circ}$, and $120^{\circ}$ elbow flexions were $38.7 \pm 4.5$ versus $40.4 \pm 3.3 \mathrm{~ms}, 38.4 \pm 3.0$ versus $39.5 \pm 2.4 \mathrm{~ms}$, and $37.8 \pm 3.0$ versus $38.4 \pm$ $3.0 \mathrm{~ms}$, respectively, showing a trend of more reduced $t_{d}$ in the patients with stroke than in the nondisabled controls and a trend of reduction in $t_{d}$ with decreasing elbow flexion (Figure 4(e)). However, neither of the reductions was significantly different $\left(F_{1,8}=1.457, p=0.26\right)$.

\section{DISCUSSION}

Reflex responses can be extremely variable, especially in patients with neurological disorders. Clinical tendon reflex scales cannot characterize the dynamic changes in large ranges and may be misleading if used on their own [12]. This study provides a useful tool for making accurate measurements of both taps to the tendon and reflex-mediated responses and characterize their dynamic relationship in terms of $G_{s}, R_{c}$, and $R_{h r}$, and reflex threshold in tapping force. The results showed markedly increased system gain, $R_{c}$, and $R_{h r}$, and decreased reflex threshold in tapping force provided quantitative measures 
to characterize hyperactive reflexes poststroke. Practically, the tendon reflex evaluation is easy to do and can potentially provide convenient and quantitative measures in clinical settings.

Spasticity is a complicated clinical symptom with multiple contributing reflex and nonreflex components. Convenient quantification tools suitable for its evaluations in clinical practice are lacking. Some investigators consider that spastic muscle hypertonus exists primarily because of an increase in reflex excitability [11,14-15]. Others argue that increased stiffness in a spastic limb could not be directly interpreted as the evidence of a hyperactive reflex because the increased resistance to a stretch could be attributed to passive tissue stiffness (from connective tissue, tendons, and passive muscle properties) and intrinsic stiffness of contracting muscle fibers, as well as reflex-mediated stiffness [16-19]. They suggest that spasticity is a complex phenomenon consisting of the reflex and nonreflex components, each of which need to be quantified related to its corresponding clinical facet for one to explain and understand the multifaceted clinical features of spasticity and to evaluate its mechanisms reliably [19-24]. Accordingly, we evaluated tendon reflexes in this study under the isometric condition, which effectively minimized the mechanical contributions of joint stiffness, viscosity, and limb inertia. Therefore, the reflex contribution was manifested and readily separated from intrinsic and passive contributions to joint torque [10,11,20-25].

This study shows that the triceps tendon reflex was much more excitable in patients with stroke than that in nondisabled controls, which was consistent with previous findings in the literature $[10,26]$. On the other hand, different from previous studies [11], this study focused on the elbow in the upper limb instead of the knee in the lower limb, and it addressed the dependence of tendon reflex on joint angles instead of at one knee position. Furthermore, this study evaluated the hyper-reflexia in a group of patients with stroke instead of in patients with multiple sclerosis, and considering different neurological disorders may involve different changes in tendon reflexes.

The $G_{S}$ was the system gain calculated through system identification relating the input of tapping forces to the output of the resultant reflex-mediated torque response. The system parameters quantified the input and output simultaneously and gave more reliable measures than did the input or output parameters alone. The increased $G_{S}$ could be caused by higher excitability of motoneurons, and it could be related to increased muscle tone poststroke so that muscle activation was converted to quicker and stronger muscle contraction. Possible neural mechanisms for spastic hypertonia in stroke included increased excitatory synaptic input and increased spindle afferent discharge rates so that motoneuronal excitability increased and excitatory postsynaptic potential from group $I_{a}$ and II muscle spindle afferents might also be enhanced related to motoneuron dysfunction. Another possible change in stroke is that inhibitory synaptic input is reduced, namely, presynaptic inhibition initiated by descending fiber input is reduced. Descending tracts could possibly contribute to spastic muscle hypertonia, either by monosynaptic excitatory projections to lower motoneurons or indirectly by inhibition of facilitation of interneurons within spinal reflex pathways.

Despite the longer contraction time and longer $R_{h r}$ associated with the higher peak of the tendon reflex torque response in the patients with stroke, the $R_{C}$ and $R_{h r}$ poststroke were significantly higher than those in nondisabled controls. The $R_{C}$ and $R_{h r}$ characterized the dynamic characteristics of tendon reflexes-how forceful and quick the reflex-mediated muscle contraction responses were. These dynamic characteristics suggest that the excitatory synaptic input such as excitation of group $I_{a}$ afferents is enhanced, while inhibitory synaptic input like group $I_{a}$ inhibitory interneurons is reduced in stroke.

There are changes of the reflex threshold in stroke. The reduced reflex threshold in tapping force could be caused by a reduced threshold in motoneuron excitability and/or peripheral changes of tighter coupling and force transmission from tendon tapping to stretching on muscle spindles poststroke. The motoneuron activity could possibly have been enhanced poststroke. The motoneuron plays a role in changing the length of the intrafusal fibers and adjusting sensitivity of the muscle spindles. In an impaired arm poststroke, muscle spindles with the inappropriate length of intrafusal fibers under all different conditions could increase group $\mathrm{I}_{\mathrm{a}}$ sensory afferent signals and decrease reflex threshold in tendon tapping force. For example, Figure $\mathbf{5}$ shows the tapping forces and peak reflex-mediated elbow extension torques on both the impaired and unimpaired limbs in a patient with stroke, which showed obviously reduced reflex threshold on the impaired side poststroke.

This study has limitations. One is that the nonreflex contribution to the increased joint stiffness poststroke was not characterized separately. Instead, the experiment 

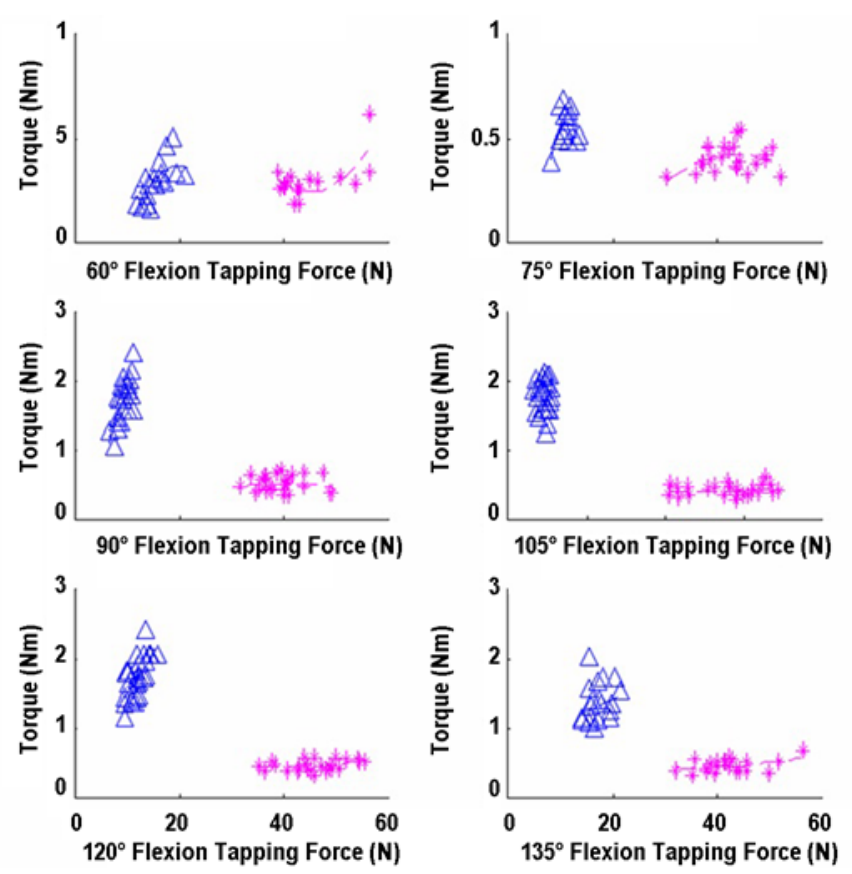

Figure 5.

Comparison of peak tapping force and peak reflex-mediated torque between impaired $(\Delta)$ and unimpaired $(*)$ upper limbs of patient with stroke at several selected elbow flexion angles $\left(60^{\circ}, 75^{\circ}, 90^{\circ}, 105^{\circ}\right.$, $120^{\circ}$, and $135^{\circ}$ ).

was conducted isometrically at the elbow joint so that nonreflex contributions were minimized. Another limitation is that the sample was small. Further study with more subjects is needed.

\section{CONCLUSIONS}

With simplification, the methods described in this article can potentially be used for clinical diagnosis and evaluations. Clinicians can potentially use them to diagnose the symptom quantitatively with higher accuracy than the clinical tendon reflex scale score of 0 to 4 . The quantitative tendon reflex evaluations would also be useful for evaluating treatment outcome more accurately. Clinicians could then track the outcome more accurately and prescribe a better treatment plan. Practically, further work must be done to simplify the setup, especially the tapping-induced output responses, and substitute them with more convenient measures, such as reflex-mediated limb movement or tendon bounce-back force [17]. This will likely make tendon reflex evaluations more suitable for the clinical setting and more conveniently performed.

\section{ACKNOWLEDGMENTS}

\section{Author Contributions:}

Acquisition, analysis, and interpretation of data: J. Liu, D. Xu, Y. Ren, L. Zhang.

Drafting of manuscript: J. Liu, D. Xu, Y. Ren, L. Zhang.

Critical revision of manuscript for important intellectual content:

J. Liu, D. Xu, Y. Ren, L. Zhang.

Statistical analysis: J. Liu, D. Xu, Y. Ren, L. Zhang.

Obtained funding: L. Zhang.

Study supervision: L. Zhang.

Financial Disclosures: The authors have declared that no competing interests exist.

Funding/Support: This material was based on work supported by the National Institutes of Health (grants R01-HD044295 and R4IN5066579), National Institute on Disability and Rehabilitation Research (grants H1335080074 and H133E100007), and National Science Foundation (grants CBET-0854498 and IIP-0750515).

Institutional Review: All subjects gave informed consent before participating in the study, which was approved by the institutional review board of Northwestern University.

Participant Follow-Up: The authors do not plan to inform participants of the publication of this study because of a lack of contact information.

\section{REFERENCES}

1. Broderick J, Brott T, Kothari R, Miller R, Khoury J, Pancioli A, Gebel J, Mills D, Minneci L, Shukla R. The Greater Cincinnati/Northern Kentucky Stroke Study: Preliminary first-ever and total incidence rates of stroke among blacks. Stroke. 1998;29(2):415-21. [PMID: 9472883$]$

2. Philip AW. Epidemiology of stroke. In: Mohr JP, Choi DW, Grotta JC, Weir B, Wolf PA, editors. Stroke: Pathophysiology, diagnosis, and management. 4th ed. Philadelphia (PA): Churchill Livingstone; 2004. p. 13-34.

3. Lance J. Symposium synopsis. In: Feldman RG, Young RR, Koella WP, editors. Spasticity: Disordered motor control. Chicago (IL): Year Book Medical Publishers; 1980. p. 485-94.

4. Bates B, Hoekelman RA, Thompson JB. A guide to physical examination and history taking. 5th ed. Philadelphia (PA): Lippincott; 1991.

5. Dietz V, Sinkjaer T. Spastic movement disorder: Impaired reflex function and altered muscle mechanics. Lancet Neurol. 2007;6(8):725-33. [PMID: 17638613]

DOI:10.1016/S1474-4422(07)70193-X

6. Bovend'Eerdt TJ, Newman M, Barker K, Dawes H, Minelli C, Wade DT. The effects of stretching in spasticity: 
A systematic review. Arch Phys Med Rehabil. 2008; 89(7):1395-1406. [PMID: 18534551$]$

7. Meythaler JM, DeVivo MJ, Hadley M. Prospective study on the use of bolus intrathecal baclofen for spastic hypertonia due to acquired brain injury. Arch Phys Med Rehabil. 1996;77(5):461-66. [PMID: 8629922] DOI:10.1016/S0003-9993(96)90034-9

8. Priebe MM, Sherwood AM, Thornby JI, Kharas NF, Markowski J. Clinical assessment of spasticity in spinal cord injury: A multidimensional problem. Arch Phys Med Rehabil. 1996;77(7):713-16. [PMID: 8670001] DOI:10.1016/S0003-9993(96)90014-3

9. Turkel CC, Bowen B, Liu J, Brin MF. Pooled analysis of the safety of botulinum toxin type $\mathrm{A}$ in the treatment of poststroke spasticity. Arch Phys Med Rehabil. 2006;87(6): 786-92. [PMID: 16731213] DOI:10.1016/j.apmr.2006.02.015

10. Zhang LQ, Huang H, Sliwa JA, Rymer WZ. System identification of tendon reflex dynamics. IEEE Trans Rehabil Eng. 1999;7(2):193-203. [PMID: 10391590] DOI:10.1109/86.769410

11. Zhang LQ, Wang G, Nishida T, Xu D, Sliwa JA, Rymer WZ. Hyperactive tendon reflexes in spastic multiple sclerosis: Measures and mechanisms of action. Arch Phys Med Rehabil. 2000;81(7):901-9. [PMID: 10896002]

DOI:10.1053/apmr.2000.5582

12. Dick JP. The deep tendon and the abdominal reflexes. J Neurol Neurosurg Psychiatry. 2003;74(2):150-53.

[PMID: 12531937]

DOI:10.1136/jnnp.74.2.150

13. Mirbagheri MM, Settle K, Harvey R, Rymer WZ. Neuromuscular abnormalities associated with spasticity of upper extremity muscles in hemiparetic stroke. J Neurophysiol. 2007;98(2):629-37. [PMID: 17537910] DOI:10.1152/jn.00049.2007

14. Rack PM, Ross HF, Thilmann AF. The ankle stretch reflexes in normal and spastic subjects. The response to sinusoidal movement. Brain. 1984;107(Pt 2):637-54.

[PMID: 6722521]

DOI:10.1093/brain/107.2.637

15. Thilmann AF, Fellows SJ, Ross HF. Biomechanical changes at the ankle joint after stroke. J Neurol Neurosurg Psychiatry. 1991;54(2):134-39. [PMID: 2019838] DOI:10.1136/jnnp.54.2.134

16. Dietz V, Berger W. Normal and impaired regulation of muscle stiffness in gait: A new hypothesis about muscle hypertonia. Exp Neurol. 1983;79(3):680-87. [PMID: 6825758] DOI:10.1016/0014-4886(83)90032-8

17. Zhang LQ, Xu D, Liao W, Rymer WZ. A quantitative and convenient method of evaluating tendon reflex and spasticity. Proceedings of the First BMES/EMBS Conference; 1999 Oct 13-16; Atlanta, GA. Los Alamitos (CA): IEEE; 1999. p. 549.
18. Lee WA, Boughton A, Rymer WZ. Absence of stretch reflex gain enhancement in voluntarily activated spastic muscle. Exp Neurol. 1987;98(2):317-35. [PMID: 3666081$]$ DOI:10.1016/0014-4886(87)90245-7

19. Sinkjaer T, Toft E, Larsen K, Andreassen S, Hansen HJ. Non-reflex and reflex mediated ankle joint stiffness in multiple sclerosis patients with spasticity. Muscle Nerve. 1993;16(1):69-76. [PMID: 8423835$]$

DOI:10.1002/mus.880160112

20. Chung SG, Van Rey E, Bai ZQ, Rymer WZ, Roth EJ, Zhang LQ. Separate quantification of reflex and nonreflex components of spastic hypertonia in chronic hemiparesis. Arch Phys Med Rehabil. 2008;89(4):700-710.

[PMID: 18374001]

DOI:10.1016/j.apmr.2007.09.051

21. Nuyens GE, De Weerdt WJ, Spaepen AJ Jr, Kiekens C, Feys HM. Reduction of spastic hypertonia during repeated passive knee movements in stroke patients. Arch Phys Med Rehabil. 2002;83(7):930-35. [PMID: 12098152] DOI:10.1053/apmr.2002.33233

22. Singer B, Dunne J, Allison G. Reflex and non-reflex elements of hypertonia in triceps surae muscles following acquired brain injury: Implications for rehabilitation. Disabil Rehabil. 2001;23(17):749-57. [PMID: 11762877] DOI:10.1080/09638280110060466

23. Zhang LQ, Chung S, Rey E, Grant T, Roth E. Reflex and nonreflex, dynamic and static changes of skeletal muscles caused by spastic hemiplegia and by intelligent stretching. Proceedings of the Banff Symposium on Skeletal Muscle; 2002; Banff, Canada.

24. Zhang LQ, Chung SG, Van Rey EM, Lin AF, Bai Z, Grant TH, Roth EJ. Evaluation and treatment of spastic hypertonia and contracture. Proceedings of the Second Joint Meeting of Engineering in Medicine and Biology. 24th Annual Conference and the Annual Fall Meeting of the Biomedical Engineering Society EMBS/BMES Conference; 2002 Oct 23-26; Houston, TX. Los Alamitos (CA): IEEE; 2002.

25. Chung SG, Van Rey EM, Bai ZQ, Rogers MW, Roth EJ, Zhang LQ. Aging-related neuromuscular changes characterized by tendon reflex system properties. Arch Phys Med Rehabil. 2005;86(2):318-27. [PMID: 15706561] DOI:10.1016/j.apmr.2004.04.048

26. Thilmann AF, Fellows SJ, Garms E. The mechanism of spastic muscle hypertonus. Variation in reflex gain over the time course of spasticity. Brain. 1991;114(Pt 1A):233-44. [PMID: 1998884]

Submitted for publication April 13, 2010. Accepted in revised form November 22, 2010.

This article and any supplementary material should be cited as follows: 
Liu J, Xu D, Ren Y, Zhang LQ. Evaluations of neuromuscular dynamics of hyperactive reflexes poststroke. J Rehabil Res Dev. 2011;48(5):577-86.

DOI:10.1682/JRRD.2010.04.0065

Crossef nimein

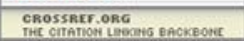


